\title{
Décadrages Décadrages
}

cinéma, à travers champs Cinéma, à travers champs

1-2| 2003

Le hors-champ

\section{Les jours où je n'existe pas: Fitoussi, le Grand Escamoteur}

Laura Legast et Marthe Porret

\section{(2) OpenEdition}

\section{Journals}

Édition électronique

URL : https://journals.openedition.org/decadrages/598

DOI : 10.4000/decadrages.598

ISSN : 2297-5977

Éditeur

Association Décadrages

Édition imprimée

Date de publication : 1 octobre 2003

Pagination : 132-136

ISSN : 2235-7823

\section{Référence électronique}

Laura Legast et Marthe Porret, «Les jours où je n'existe pas : Fitoussi, le Grand Escamoteur », Décadrages

[En ligne], 1-2 | 2003, mis en ligne le 26 avril 2013, consulté le 26 mars 2022. URL : http://

journals.openedition.org/decadrages/598 ; DOI : https://doi.org/10.4000/decadrages.598

(B) Décadrages 


\section{Les jours où je n'existe pas: Fitoussi, le Grand Escamoteur}

par Laura Legast et Marthe Porret

Entretien effectué à Genève le 22 février 2003 à l'occasion de la projection des Jours où je n'existe pas au cinéma Spoutnik, en présence du réalisateur.

Né le $1^{\text {er }}$ juin 1970 à Tours, Jean-Charles Fitoussi étudie les arts plastiques, la philosophie et l'architecture avant de réaliser en 1994 le moyen métrage Aura été, son premier film, grâce à une aide de la Fémis sous forme de matériel. Suite à un travail d'assistanat sur le film de Christian Merlhiot, Les semeurs de peste, tourné en 1995 à la Villa Médicis, Fitoussi tourne son premier long métrage, D'ici là, à Rome. C'est dans cette ville qu'il rencontre J.-M. Straub et D. Huillet et qu'il devient leur assistant de 1996 (Von Heute auf Morgen) à 2002 (Umiliati!). Après avoir réalisé en 2001 Sicilia! Si gira, documentaire sur la fabrication du film de Huillet et Straub Sicilia!, il termine en 2002 son deuxième long métrage: Les jours où je n'existe pas. Le film met en scène Antoine, un homme qui ne vit qu'un jour sur deux. À minuit, il disparaît soudainement pour réapparaître vingt-quatre heures plus tard au même endroit. Reclus dans son appartement, il s'accommode tant bien que mal de son inavouable handicap jusqu'au jour où il rencontre Clémentine, vivante «à plein temps»...

Votre film, qui raconte l'bistoire d'un personnage ne vivant qu'un jour sur deux, ne cesse de faire référence à un hors-champ imaginaire, un espace-temps imperceptible. Quel intérêt portez-vous à cette notion de bors-champ?

Le hors-champ appartient à l'essence même du cinéma, conçu non seulement comme un art de voir et d'entendre, mais aussi comme un art de la suggestion et de l'imaginaire. À partir du moment où l'on décide de faire un film, on réfléchit nécessairement à cette notion-là, puisque faire un cadre, c'est définir un hors-champ. Il y a chez moi une volonté d'évoquer une forme de totalité du monde, et du temps. Totalité qu'il est évidemment impossible de montrer, mais qui peut être rendue sensible par la si forte présence que l'on peut donner au hors-champ cinématographique. Le hors-champ dilate l'espace et le temps en nous faisant ressentir combien le champ 
n'est qu'une découpe d'un ensemble plus vaste. C'est d'ailleurs en quoi le découpage ne détruit pas le réel, cette «robe sans couture» dont parlait Bazin, mais en révèle l'étendue. La coupe et le horschamp mettent l'accent a contrario sur l'absence de coupe et de hors-champ du monde perçu dans sa totalité, d'un point de vue divin. Il y a longtemps que je m'intéresse à ce moment de la coupe dans laquelle disparaît beaucoup de temps, de réalité non-filmée. J'ai toujours eu une jouissance très forte à percevoir cet instant magique, trace de disparition. C'est une émotion intense de percevoir un raccord au cinéma. Dès l'écriture, puis encore au tournage, je pense sans cesse aux raccords. Chaque fois que je finis un plan, j'imagine le moment de la coupe, le rapport plastique qui va s'opérer entre ce plan et le suivant. Parfois, bien sûr, on trouve d'autres rapports au montage. Mais c'est de toute façon une préoccupation permanente.

\section{Votre personnage principal est très "cinématogra-} phique», il vit grâce aux raccords.

À l'origine, ce personnage qui existe par intermittence a été conçu par l'écrivain états-unien Nathaniel Hawthorne. Il m'est tout de suite apparu que sa vie était à l'image de ce qu'est un film. Un film vit par intermittence, chaque plan étant un moment d'existence, suivi d'une coupure d'inexistence. Si dans un film les ellipses entre les séquences sont perçues comme telles, on s'efforce en revanche la plupart du temps de rendre imperceptible les "micro-ellipses" entre chaque plan. Or ces ellipses-là, d'un plan à un autre, m'intéressent énormément. De même qu'un spectateur n'a en général pas conscience des trous entre chaque plan, Antoine n'a pas conscience du passage entre deux jours où il existe, puisque entretemps il appartient au néant. Hollywood a réussi à rendre invisible ces raccords et à redonner une sensation de continuum. Dans mon film, j’ai joué sur l'idée de continuum en utilisant moi-même un découpage à peu près classique, tout en laissant au spectateur la perception du raccord, et ce par différents moyens: en utilisant le raccord pour faire disparaitre des personnages, en tournant en son direct intégral, en ne cherchant à masquer ni les variations de lumière ni celles de son entre chaque plan. Ce qui engendre une tension entre d'une part un découpage classique et de l'autre des éléments visuels et sonores qui laissent se manifester cet incident, cet accident qui est le raccord. Il faut rappeler que le tournage s'est étalé sur deux ans. On a donc raccordé des blocs d'existence qui étaient énormément éloignés à la fois par l'espace et par le temps. Pour la séquence de la barque qui dure quelques minutes dans le film, le tournage s'est étalé sur une journée entière. Entre le début de la séquence et la fin, on passe du matin à la nuit. Les ruptures d'un plan à l'autre sont très sensibles, à mesure que la lumière décroît: le temps semble s'accélérer, et passe pour le spectateur exactement comme pour Antoine. Ce qui crée, pour le coup, une espèce d'étrangeté. Même si l'attention d'un spectateur n'est pas exercée à percevoir ces choses-là, je pense qu'elles passent de manière plus ou moins inconsciente, et qu'on doit sentir, si infimes soient-elles, les failles entre chaque plan, même dans les séquences découpées de manière classique. Cela devrait provoquer comme une légère dissonance en musique. À cet égard, j’aime aussi entendre les changements d'ambiance dans un raccord, ces micro-variations qui constituent une sorte de musique.

Evoquons la première "disparition" du film, celle de la petite fille Laure devant le miroir, qui se révèle n'être finalement qu'une ellipse. En dépit de cette justification temporelle, le changement de lumière et le nouveau positionnement de Laure dans le cadre provoquent après la coupe un effet d'étrangeté.

Le début est une forme de mise en jambe, de prologue, d'introduction au thème et aux motifs du film. Mais, effectivement, il y a une ellipse temporelle, ce qui fait qu'il est naturel de ne plus retrouver le personnage à cet endroit. Simplement, on a fait attention à conserver exactement le même cadre: la lumière change alors que les lignes demeurent et la jeune fille 
n'est plus là. On la retrouve à la fin du panoramique dans son lit. Mais en fait, je ne fais qu'utiliser le cinéma de manière habituelle! Disons que l'étrangeté de l'ellipse temporelle, sa bizarrerie, est révélée. Si le second plan avait commencé directement sur la jeune fille dans son lit, le spectateur n'aurait ressenti aucune disparition. En commençant sur la portion d'espace où elle était, on met l'accent sur le fait qu'elle n'y est plus, qu'elle a en quelque sorte cessé d'exister à cet endroit: on filme alors une absence.

\section{La deuxième disparition, la mort du vieillard, fait inter- venir une autre dimension...}

Oui. Il s'agit là non plus seulement de disparaître d'un endroit, mais de tous les endroits. Le film participe d'une interrogation sur l'existence et le néant. Je me suis souvent posé la question de savoir quelle différence il y avait entre une personne très lointaine, avec laquelle on n'a plus de contact, et une personne morte. Dans les faits, pour celui qui n'a plus de nouvelles, c'est la même chose. À ceci près, qui est capital: un vivant avec qui vous n'avez aucun contact garde le potentiel de se manifester, et surtout se manifeste à d'autres. Il m'est arrivé d'apprendre tardivement la mort de quelqu'un que je n'avais pas vu depuis plusieurs mois. Rétrospectivement, ce que je croyais être un temps habité par cette "présence lointaine» devint un temps dépeuplé. On croyait le monde occupé à un certain endroit par cet être, et l'on réalise que depuis déjà un certain temps cet être n'était plus là pour personne. La nature de ce temps s'en trouve changée, et le choc est aussi grand que si vous aviez été contemporain de sa mort. Je voulais placer cette histoire un peu fantaisiste dans le cadre de cette interrogation sur le néant, confronter ces disparitions provisoires à la disparition radicale dans la mort. S'ajoute un autre questionnement: quelle est la différence entre n'avoir jamais existé et avoir cessé d'exister? Jankélévitch ${ }^{\mathbf{1}}$ pose cette question, et souligne que ce qui a été ne peut plus ne pas avoir été. Le fait d'avoir existé est à jamais ineffaçable, et constitue notre forme d'éternité.
C'est seulement après ces deux premières "disparitions" que le spectateur assiste enfin à celle d'Antoine. Comment l'avez-vous mise en scène?

Antoine se couche peu avant minuit et s'endort / Changement d'axe: Antoine ne figure plus dans le plan / Retour à l'axe premier: Antoine est présent dans le champ, le premier plan se poursuit. Là, on est dans un point de vue objectif, extérieur à Antoine: on le voit disparaitre, puis réapparaître. Il s'est donc écoulé vingt-quatre heures. Deux solutions étaient possibles pour rendre compte de ce point de vue extérieur: ou bien recourir à l'effet magique de Méliès, c'est-à-dire interrompre l'enregistrement pour permettre au personnage de quitter le champ en maintenant fixe la caméra, ou bien explorer toutes les autres possibilités. Je n'avais pas envie de recourir à l'effet-Méliès, un peu usé, qui m’apparaissait comme une facilité, dont on ne perçoit plus tellement aujourd'hui la dimension fantastique. Le jeu était de faire disparaître quelqu'un sans jamais recourir à l'effet-Méliès.

Mais pourquoi avoir modifié l'axe de la caméra? Cette coupe, avec changement d'axe, pourrait être une simple ellipse temporelle et pas forcément une disparition fantastique.

C'est alors le commentaire qui précise qu'il disparaît à minuit, en plus de l'effet visuel qui manifeste la disparition.

Antoine disparaît plusieurs fois au cours du film. Qu'enest-il de sa deuxième disparition?

Elle a lieu quand il est dit que bien souvent Antoine avait attendu minuit tout éveillé pour savoir ce qui se passait. Dans cette séquence, Antoine regarde sa montre et je fais le raccord le plus invisible qui soit, un raccord dans l'axe et dans le mouvement : ainsi les spectateurs ne perçoivent pas la coupe. Il s'agit ici de

1 En hommage au philosophe français Vladimir Jankélévitch (Bourges, 1903-Paris, 1985), Les Jours où je n'existe pas s'ouvre sur une plaque commémorative de la ville de Paris citant un passage de son œuvre. 
placer le spectateur exactement dans le point de vue, dans la perception d'Antoine qui attend le coup de minuit et voit qu'il ne se passe rien. Il n'a pas senti passer ses vingt-quatre heures de néant.

Pourquoi n'avoir pas plutôt choisi de faire un planséquence sans coupe?

Ah non! Il faut que cette coupe existe, puisque vingtquatre heures s'y sont engouffrées. C'est là le principe même de son existence: Antoine, comme je l'ai dit, est exactement à l'image d'un film. Les jours où il n'existe pas coïncident avec un arrêt de la caméra, un raccord. Dans cette deuxième disparition d'Antoine la coupe existe mais n'est pas perçue. Mais il faut qu'elle existe! Le problème de la vie d'Antoine est justement un problème de raccord. Tant qu'il vit seul dans son appartement où rien ne bouge, ses jours raccordent parfaitement. Peut-être un peu de poussière s'est-elle accumulée. Mais lorsque Clémentine entre dans sa vie, elle qui vit à plein-temps, le trouble d'Antoine provient de ce qu'elle finit par ne plus faire le raccord, lorsqu'elle rentre en retard.

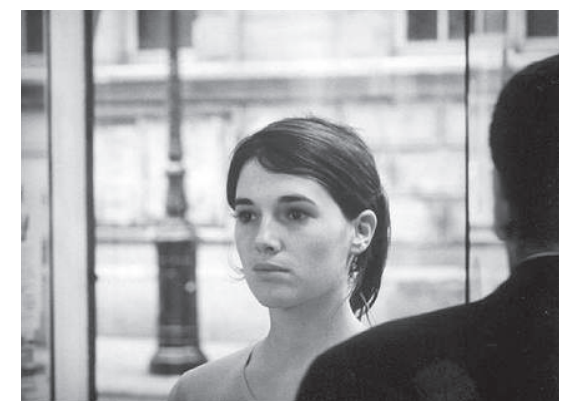

Plus le film avance, plus vous faites confiance au spectateur pour comprendre tous ces jeux de disparitions et de réapparitions. Il y a une progression.

Oui, il y a même un moment où l'on occupe vraiment le point de vue d'Antoine et où le spectateur participe à sa stupéfaction. La séquence de la barque se termine à la tombée de la nuit, Antoine a hâte de rentrer par peur de disparaître dans un endroit inconnu. La séquence suivante commence sur Antoine, dans son lit, parlant à Clémentine hors-champ. Le spectateur pense qu'il s'agit toujours de la journée de promenade en barque. Antoine croit qu'il s'adresse à Clémentine, mais elle ne répond pas. C'est dans l'absence de sa réponse qu'il se rend compte qu'elle n'est plus là et qu'il s'est donc écoulé vingt-quatre heures. Le spectateur est lui-même surpris, puisque cette fois la coupe n'a pas eu lieu au sein même de la séquence, mais entre la séquence de la barque et celle de l'appartement. Antoine dans leur couple était donc en train de parler tout seul. Le spectateur, comme Antoine, croyait à la présence de Clémentine hors-champ, y croyait sans pourtant la percevoir, il peuplait luimême le hors-champ, par habitude.

Dans votre film plusieurs éléments restent en suspens. Le jeune Antoine et Antoine adulte sont-il une seule et même personne? Laure, l'amie d'Antoine, ressemble étrangement à Clémentine, cette ressemblance est-elle voulue? Quelle relation faut-il établir entre le vieillard qui meurt au début du film et Antoine?

Je laisse le spectateur libre d'imaginer ce qu'il veut. On peut penser que ce vieillard est son père, ou son grand-père - c'est un vieil homme qui meurt. Pour les prénoms, il y a une part de hasard. Je voulais garder, par principe, le prénom des acteurs eux-mêmes: les modifier, c'est-à-dire inventer des prénoms de fiction, aurait ajouté une intention signifiante et je préférais ne pas intervenir là-dessus. Il s'est trouvé que les deux acteurs principaux s'appelaient Antoine. C'est un hasard, mais comme tous les hasards, il devient une nécessité dans le film, puisque ces deux personnages vont être liés d'une certaine manière. Antoine Chappey m'a confié, pendant le tournage, qu'il avait toujours perçu la part de négation que contenait son prénom, entendu comme «en toi ne», voire "en toi noud». Et puis Chappey évoque «échapper». Voilà pour les influences lacaniennes! Par ailleurs, quand j'ai cherché l'amie de l'enfant et que j'ai trouvé Laure, j’ai été frappé et séduit par cette ressemblance avec Clémentine. Je suis très intéressé 
par les notions de répétition, de retour à l'identique. Et ces ressemblances et homonymies suggèrent un tel retour éternel.

Quel effet le temps a-t-il sur Antoine? À quel rythme vieillit-il? Meurt-il?

Il vieillit deux fois moins vite, puisque pendant son passage dans le néant, il ne vieillit absolument pas : il n'existe pas! Il est né en 1920, il a donc quarante ans en 2000. Je ne montre pas la mort d'Antoine - qui d'ailleurs ne meurt pas vraiment. Pour moi, la mort se passe toujours dans un hors-champ, il en est ainsi de celle du vieil homme. On peut observer un mourant, on ne saura qu'il est mort qu'après-coup! Là encore, Jankélévitch a consacré de belles pages au fait qu'on ne sait jamais quand a lieu le dernier soupir, le dernier souffle. On ne voit jamais l'instant de la mort. Ce dernier instant est à jamais imperceptible pour un vivant. On ne peut jamais localiser cet instant dans le temps. Même avec un encéphalogramme, ce n'est qu'à partir d'un certain temps, quand il n'y a plus d'impulsions, que l'on se rend compte de la mort: on observe les battements du cœur, et on ne sait jamais si celui que l'on vient de voir sera le dernier. Il faut attendre. S'il n'en vient plus d'autres, alors le précédent aura été le dernier. Il n'est perçu comme le dernier instant qu'après coup : sur le moment, on ne sait rien.
Pourquoi avoir choisi Antoine Chappey pour le rôle principal?

Je l'ai vu dans des films. Et ce qui m'est apparu, c'est qu'il avait une présence particulière. Il fait partie de ces acteurs que l'on n'oublie pas, et pourtant qu'on est incapable de vraiment bien fixer. Il est là sans l'être tout à fait. Il a une présence paradoxale. Même quand il est au premier plan, il reste d'une certaine manière au second. Chappey échappe, dirait Lacan. Que dire d'autre si ce n'est qu'il me paraissait convenir à merveille pour ce personnage, mi-présent, mi-absent?
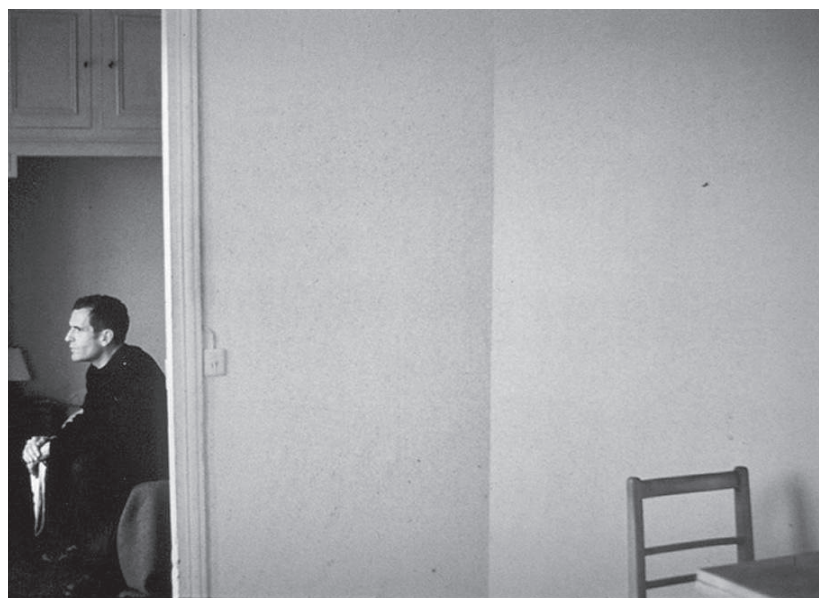

Les jours où je n'existe pas (France, 2002, 35 mm, $110 \mathrm{~min}$.

Présenté au Festival de Locarno 2002 (Cinéastes du présent).

Réalisation et scénario: Jean-Charles Fitoussi. Image: Céline Bozon, Thierry Taïeb, Aurélien Devaux. Montage: Pauline Gaillard. Son: Erwan Kerzanet, Yolande Decarsin. Interprétation: Antoine Chappey, Luis Miguel Cintra, Clémentine Baert, Antoine Michot, Jean-Paul Bonnaire, Serge Bozon, Yves Caumon, Claire Doyon et Helmut Färber. Production: Nathalie Eybrard, Jean-Philippe Labadie, Jean-Charles Fitoussi. 